\title{
Simulation of Simplified BSPD Deploying Timer Circuit for a Prototype Formula Electric Car
}

\author{
Benny Paul, V. Preethi, T. V. Narmadha, K. Arun
}

\begin{abstract}
Now-a-days electrical vehicles are growing faster than the vehicles fuelled with conventional energy resources and are expected to replace conventional vehicles in near future,owing to its zilch emissions and environmental friendly. Albeit the electrical vehicles are contributing to the reduction in air and noise pollution, few technical issues have to be resolved for its improved performance. Currently electric vehicle is turned off automatically, for avoiding the shaft failure due to the twisting load acting on the shaft, while operator applies the accelerator pedal and brake pedal simultaneously. Hence, an attempt had been made to delay the system getting turned off by introducing a timer circuit in BSPD (Brake System Plausibility Device). The functioning of simplified BSPD with timer circuit was simulated using the Proteus software andthe outcome of the simulated results is presented in waveforms, which confirms the proper functioning of the circuit. $A$ PCB for engaging the shutdown circuitwas also developed to control the electric vehicle's powertrain. The hardware implementation of the simulated circuit had been carried out and the real time working of the circuit was verified.
\end{abstract}

Keywords-Electric vehicles, Timer, Electronic throttle, Brake pedal, BSPD

\section{INTRODUCTION}

Increased population and rapid industrialization depend mainly on road transport, leading to substantial growth of automobile sector, resulting in anincreased consumption of conventional fuels. Higher consumption of conventional fuels affects the air quality leading to the emission of green house gases. Internal combustion engines are heavier; convert the reciprocating motion of piston into the rotary movement that incur mechanical loses due to changes between directions of motions; produce usable torque only in a narrow band of engine speed.

Electric vehicles could perform more effectively compared to internal combustion engines as it overcomes all the above limitations. Thus utilization of electric vehicles would be a betteralternativefor ensuring sustainable environment and to reduce the shortage of energy demand in the future.Electrical vehicles shall be fault tolerant, reliable and guarantee the required safety. Although electrical vehicles are commercialized, intensive research is still in progress and safety has to be ensured during its operation.
Electric power train is getting damaged owing to the excessive negative torque developed and leads to shaft failure as result of fatigue loading, due to frequent braking while the motor is powered [9]. For preventing the shaft failure, controlling of either hard braking or motor powering is essential. Since hard braking play a vital role in bringing the vehicle immediately or in short interval of time at an emergency/critical situation, limiting/controlling braking power is not safe.

From the literatures, it is observed that the braking control with wheel speed sensor and acceleration sensoris ineffective and not precise to develop a fault code [7]. Plausibility check devices, used in various applications,recognise faults quickly and execute a function safely.BSPD is one among the device that controls shutting down the motor, in case of simultaneous motor powering and hard braking, ensuring the driver safety by opening the tractive system.BSPD is non-programmable electronically controlled plausibility check device which disconnects the motor from its power source with greater accuracy and precision.

From the above discussion, it was decided to develop timer circuit with BSPD for delaying the shutdown of an electric vehicle. It was also intended to simulate the developed circuit using Proteus software and to present the simulated results to ensure the functioning of the circuit.

Revised Manuscript Received on December 5, 2019.

Mr. Benny PaulI, Student, B.E. Mechanical Engineering, St. Joseph's College of Engineering, Chennai, India.

Ms. Preethi V, Student, B.E. Electrical and Electronics Engineering, St. Joseph's College of Engineering, Chennai, India.

T. V. Narmadha, Professor, Department of Electrical and Electronics Engineering, St. Joseph's College of Engineering, Chennai, India.

Dr. K. Arun, Associate Professor, Department of Mechanical Engineering, St. Joseph's College of Engineering, Chennai, India. 


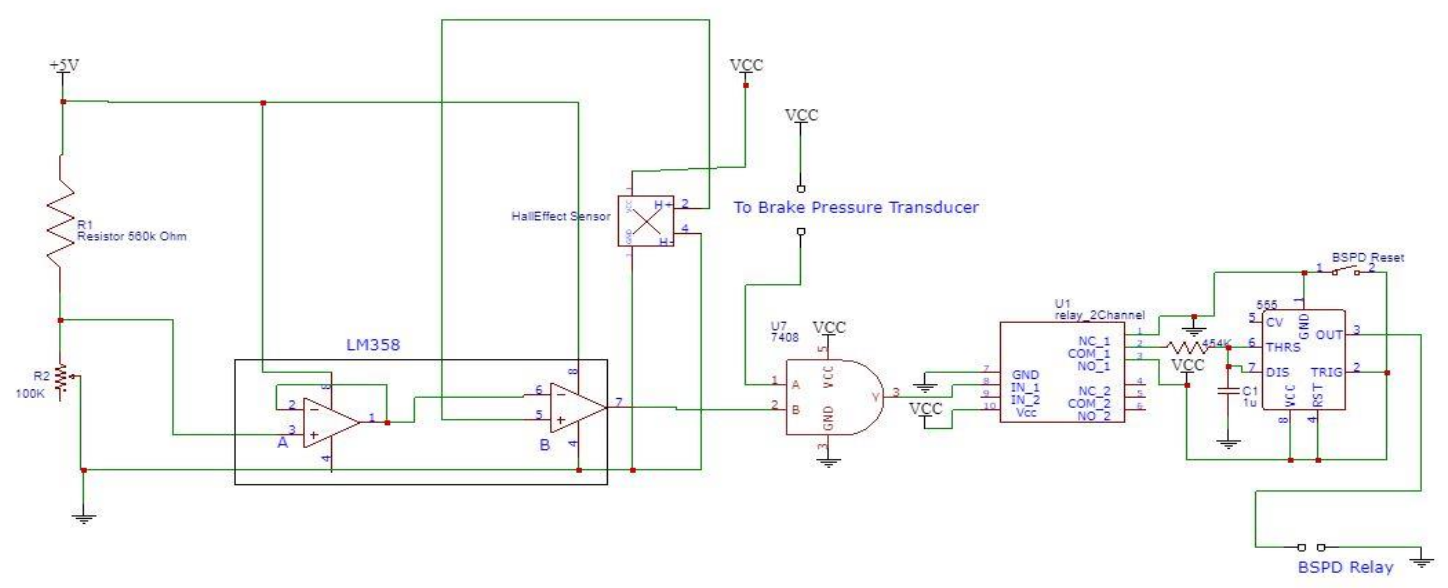

Figure 1 Circuit Diagram of BSPD

\section{EXPERIMENTAL SETUP}

The BSPD circuitalong with its components and its functioning isshown in figure 1 and is described below in detail.

The signal from the Hall Effect base current sensor and brake pressure sensor is taken as key input to the circuit. Output signal from the Hall Effect sensor is given to the comparator block against a reference value of $5 \mathrm{~kW}$ (i.e. $60 \mathrm{~A}$ for 144V). The comparator block consists of IC LM358, 550 $\mathrm{k} \Omega$ resistor and $100 \mathrm{k}$ of potentiometer. A $5 \mathrm{~V}$ supply was given to the pin 1 of the op-amp comparator (LM358). The reference value of $1.2 \mathrm{~V}$ is given as an input to the noninverting terminal of the comparator 1 through a potential divider as we get an output of $1.4 \mathrm{~V}$ from the Hall Effect sensor for $60 \mathrm{~A}$. The comparator 1 is used as a unity gain comparator to reduce noise level. The output from unity gain comparator is given to the inverting input of comparator 2. This reference value is compared with the output voltage from the Hall Effect sensor which is given to pin 3 (non-inverting terminal) of the comparator 2 . If the voltage value goes higher than the reference voltage, the comparator outputs a signal to the trigger block.

Trigger block comprises 4 bar brake pressure sensor, IC 7408 and single channel relay. The brake pressure sensor measures the oil pressure in the brake lining and produces a signal if it reaches a value of 4 bar as an indication of hard braking. The output signal is given to an AND gate (IC 7408). If the output from the comparator and the brake pressure sensor is high then it inputs a signal to the single channel relay. This causes the relay to change its position from normally open side to normally closed side triggering the timer circuit.

A timer value of $500 \mathrm{~ms}$ is set as reaction time for accidental simultaneous pressing. A separate $12 \mathrm{~V}$ supply is given to the timer by the trigger block. The output of the timer is given as supply to the shut-down relay. The reset pin of the timer (pin 4) is given active low signal using a switch which grounds the reset pin only when it is pressed.

Current drawn from the motor is measured using a current sensor, which converts current to an easily measured output voltage. Current sensors are used in various applications such as space [1], hybrid vehicles. Hall Effect based current sensor is one among various different current sensors available. Hall Effect current sensors provide lossless and isolated current sensing solution, by sensing the current without dissipating the power that passive resistors do [1]. Such magnetic sensors, integrated in regular CMOS technology, prove to be cost-effective and offer high performance [2]. The circuit comprises of two Hall Effect based current sensor with an open core magnetic concentrator for good reliability and repeatability [3]. An average value of output from two Hall Effect sensors is taken for Redundancy based plausibility checking as it provides a much higher potential in terms of detection time and precision [4] which is givento a comparator. This comparator compares it against a reference value. If the output from the comparator block and a brake pressure sensor is high the trigger block sends signal to a timer circuit. After a pre-set amount of time, the timer block sends a signal to the relay and thereby opening the shutdown circuit.

\section{SIMULATION OF BSPD}

The BSPD with timer circuit was designed and simulated in Proteus software for obtaining timing diagram and the functioning of circuit was visualised in a virtual oscilloscope. The outcome of the simulated results is shown in the form of timing graph. The simultaneous operations are obtained in the form of two timing diagrams and are presented in the figures 4 and 5 .

Hall Effect sensor and brake pressure sensor inputs signal to the trigger block when the brake pedal has been pressed simultaneously while the motor was also powered. The trigger block triggers the timer circuit by supplying a voltage to pin 6 (Threshold pin). The 555 timer is connected in monostable mode. The timer is designed in such a way that if it is triggered, then after the pre-set amount of time supply to the shut-down relay is cut and once it has been triggered it needs an external reset switch to turn it on again.

A $5 \mathrm{~V}$ supply is given to power the circuit. Two oscilloscopes are used to obtain timing diagrams from the AND gate and timer blocks. 


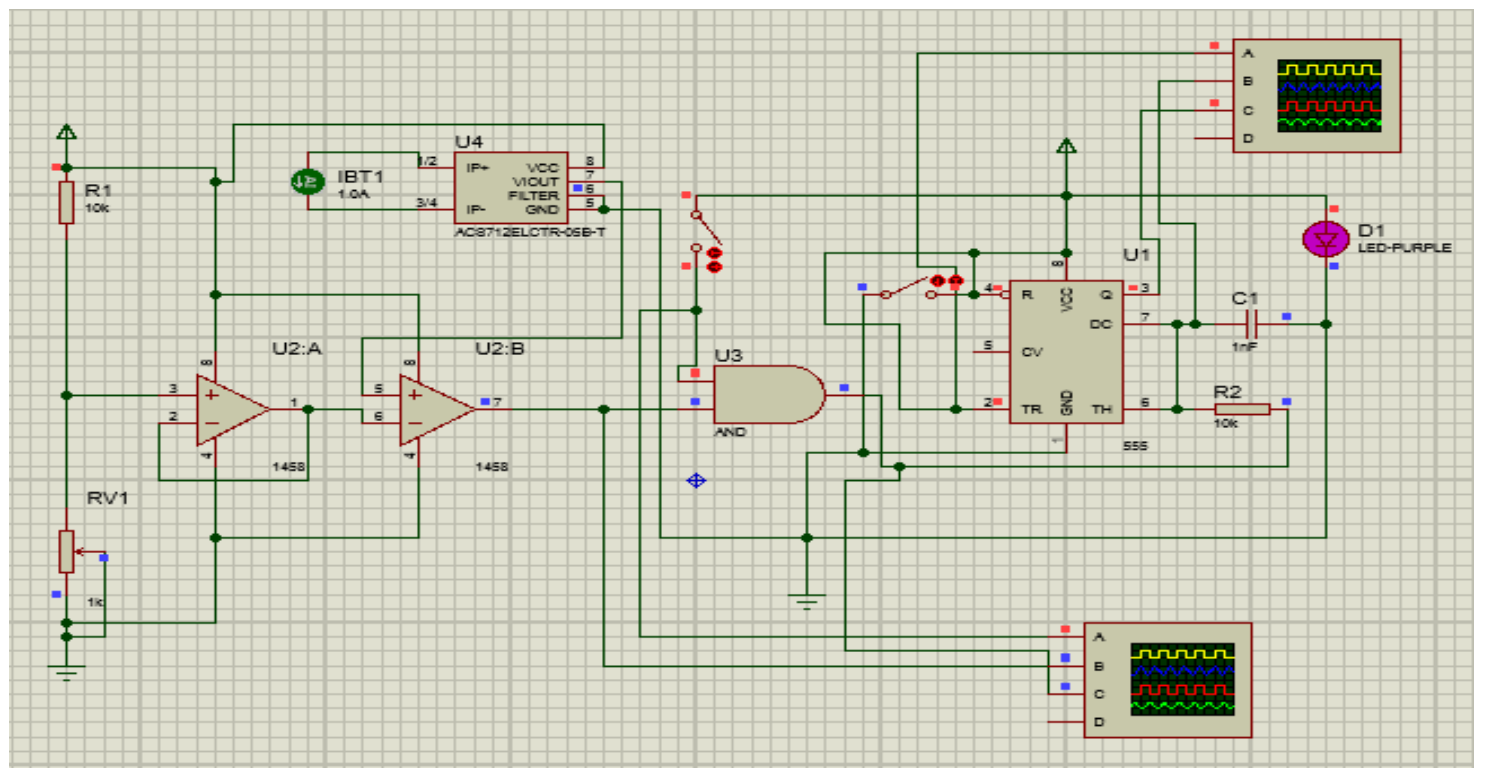

Fig.2.Simulation setup of entire Circuit

\section{RESULTS AND DISCUSSION}

Output voltage from the Hall Effect sensor was measured at regular intervals for respective current input. A reference voltage was initially set for 60A current input which was compared with the output from the Hall Effect sensor using a comparator. A graph was drawn against voltage and current using the measured values.

From the figure 3, it was observed that the output voltage from the hall effect sensor increases linearly as the current through the load increases. The linearly rising graph intersects the reference voltage (horizontal) at 60A.Subsequently, comparator block sends a continuous signal to the AND Gate as shown in the below timing diagram. Since signal is continuous, one of the inputs of the AND Gate was always high.

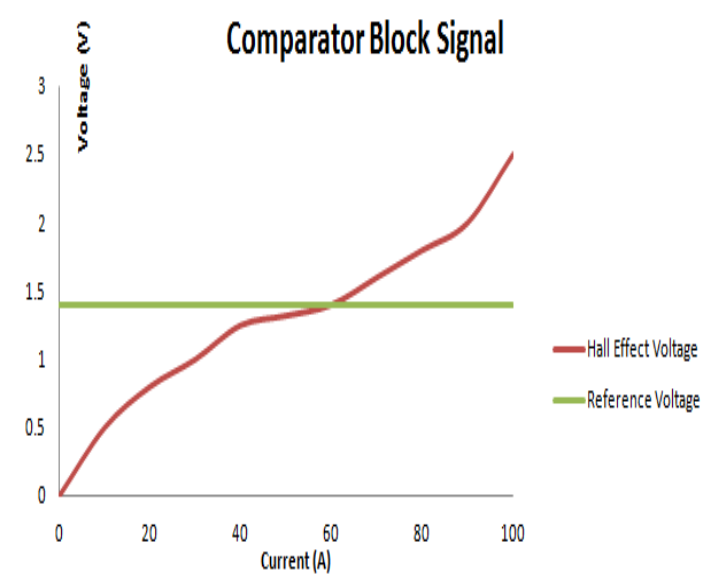

Fig.3. Comparator Block Signal

The figure 4 depicts the status of the brake pressure sensor. It sends a high signal to the AND gate when it senses a hard braking pressure of 4 bar and hence output goes high as shown in the last line of the timing diagram.

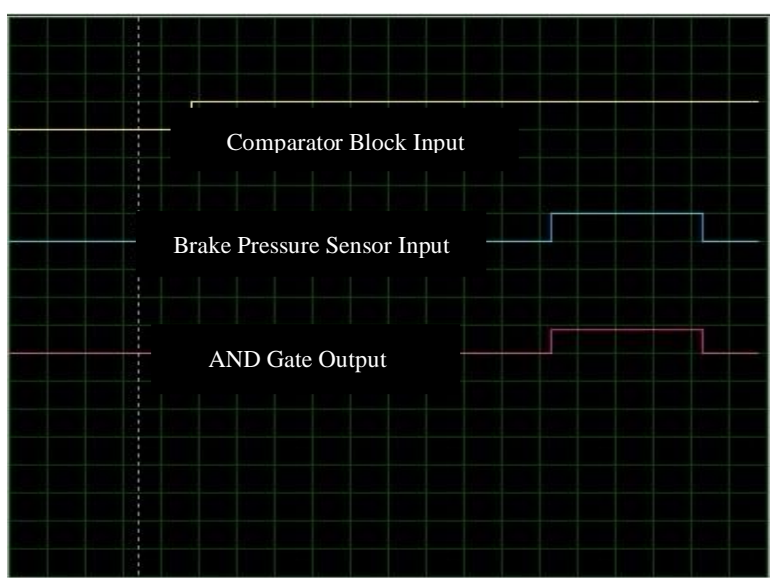

Fig.4. Timing Diagram of AND Gate

Initially the trigger pin of the timer is given an active low signal to make the output highas shown in the first line in figure 5. When the signal from the AND gate reaches the timer block and the timer starts and makes the output go low after a pre-set amount of time as shown in the second and third timing line turning off the shutdown circuit.

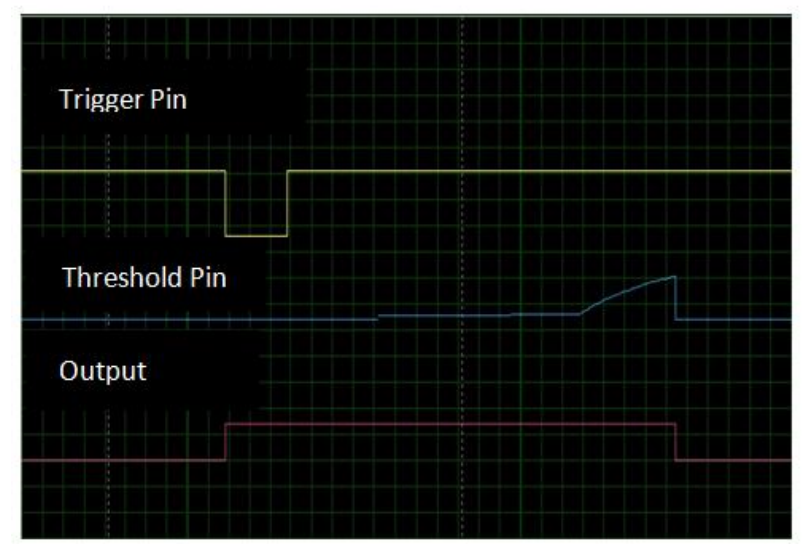

Fig.5. Timing Diagram of Timer Circuit 


\section{CONCLUSION}

The simplified BSPD with timer circuit for delaying the shutting down of the electric vehicle has been developed and simulated using Proteus software.

Outcome of the simulated results revealed that the developed circuit functions welland avoids mechanical failure during powering. The non programmable circuit ensures avoiding any form of programmable errors that cannot be resolved by layman and also it is evident that simplicity of the circuit makes it easy to reassemble if any failure occurs.

\section{REFERENCES}

1. "Analysis of Different Hall Effect Current Sensors for Space Applications", Arya Krishna,

Lizy Abraham, International Journal of Innovative Science, Engineering \& Technology, Vol. 1 Issue 5, July 2014.

2. "Hall Effect Sensors Design, Integration and Behaviour Analysis", Maria-Alexandra Paun, Jean-Michel Sallese and Maher Kayal, February 2013.

3. "Modelling of a Hall Effect-Based Current Sensor with an Open Core Magnetic Concentrator", Ivan Yatchev, Mehmet Sen, IoskoBalabozov and Ivan Kostov, April 2018.

4. "Plausibility Checking of Sensor Signals for Vehicle Dynamics Control Systems", Heiner Versmold, Martin Saeger,

5. "The Design, Implementation, Evaluation and Results of a Race Car for the Collegiate Formula SAE Electric Competition", Quinn Jasha Bryan Sullivan,7 December 2016.

6. "Brake Pressure Control Method and Apparatus, Including a Brake Pressure Booster", Helmut Heibel, 27 June 2010

7. "Plausibility Checks in Automotive Electronic Control Unit to enhance Safety and Security", Martin Ring, Reiner Kriesten, September 2016

8. "The Electrical Circuitry for Formula SAE- Electric 2012", Thomas Braunl, 2 November 2012

9. "Fatigue Analysis of Drive Shaft", Sandeep Gujaran, ShivajiGholap, October 2014

\section{AUTHORS PROFILE}

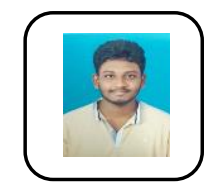

Mr. Benny PaulI is the student of St. Joseph's College of Engineering, Chennai, India, pursuing B.E. Mechanical Engineering. His areas of interests and projects were on conservation of energy and automation industry. Specifically, he has worked on projects in the fields related toAir Conditioning, E-Car and Hydraulics. He was also a studentmember of ISHRAE for 2 years.

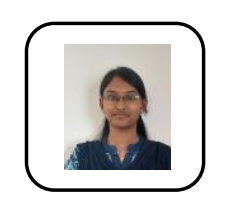

Ms. Preethi V is the student of St. Joseph's College of Engineering, Chennai, India, pursuing B.E. Electrical and Electronics Engineering. Her areas of interests are Renewable Energy and DC Electrical Machines. She has worked on projects such as Wind power uncertainty analysis using particle swarm optimisation and E-Car. She was also a member of IEEE.

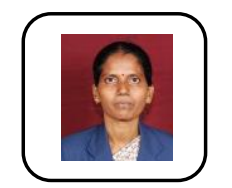

T. V. Narmadhais a Professor in the Department of Electrical and Electronics Engineering, St. Joseph's College ofEngineering, Chennai, India. She obtained her AMIE(EEE) from the Institution of Engineers, India and M.E., and PhD degree from College of Engineering, AnnaUniversity, Chennai. She has more than 20 years of teachingexperience. She has published more than 25 research papers in national/international journals/conference proceedings.Her teaching and research interests include electricaldrives, auto tuning, control using fuzzy logic, neuralnetworks and genetic algorithm. She is a Associate member of IE (I).

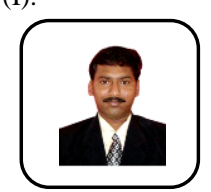

Dr. K. Arun is an Associate Professor in the Department of Mechanical Engineering, St. Joseph's College of Engineering, Chennai, India. He completed B.E. Mechanical Engineering from
Bharathidasan University, Tiruchirappalli with First Class and M.E. in Energy Engineering from Institute for Energy Studies, College of Engineering Guindy Campus, Anna University, Chennai with First Class Distinction. He has been awarded with Degree of Doctor of Philosophy from Faculty of Mechanical Engineering, Anna University, Chennai. He has around 20 years of teaching experience and published around 10 research papers in national/international journals/conference proceedings. His areas of specialization include Biomass Gasification, Energy Conservation, Renewable Energy Technologies, Fluid Heat Transfer Analysis and Waste Heat Recovery Systems. He is an active reviewer in Elsevier, Taylor \& Francis and American Institute of Physics Publishing Journals and has been awarded with certificate for the Outstanding Contribution in Reviewing Manuscripts by Elsevier. He was the Guest Faculty in the Department of Mechanical Engineering, College of Engineering Guindy Campus, Anna University Chennai. He had also presented technical sessions in AICTE sponsored Short Term Training Programme and Faculty Development Programmes sponsored by Anna University. He is a member of Staff Selection Board in Chendhuran College of Engineering and Technology, Pudukkottai. 